\title{
Pupil Detection Based on Color Difference and Circular Hough Transform
}

\author{
Hazim G. Daway ${ }^{1}$, Hana H. kareem ${ }^{2}$, Ahmed Rafid Hashim ${ }^{3}$ \\ ${ }^{1}$ Department of physics College of Science, Mustansiriyah University, Iraq \\ ${ }^{2}$ Department of physics, College of Education, Mustansiriyah University, Iraq \\ ${ }^{3}$ Department of Computer Science, College of Education for Pure Sciences-Ibn Al- Haitham, University of Baghdad, Iraq
}

\begin{tabular}{l} 
Article Info \\
\hline Article history: \\
Received Jul 23, 2017 \\
Revised Jan 20, 2018 \\
Accepted Feb 11, 2018 \\
\hline
\end{tabular}

\section{Keyword:}

Binary image

Circular hough transform

Color difference

Iris detection

Pupil area

\begin{abstract}
Human pupil eye detection is a significant stage in iris segmentation which is representing one of the most important steps in iris recognition. In this paper, we present a new method of highly accurate pupil detection. This method is consisting of many steps to detect the boundary of the pupil. First, the read eye image (R, G, B), then determine the work area which is consist of many steps to detect the boundary of the pupil. The determination of the work area contains many circles which are larger than pupil region. The work area is necessary to determine pupil region and neighborhood regions afterward the difference in color and intensity between pupil region and surrounding area is utilized, where the pupil region has color and intensity less than surrounding area. After the process of detecting pupil region many steps on the resulting image is applied in order to concentrate the pupil region and delete the others regions by using many methods such as dilation, erosion, canny filter, circle hough transforms to detect pupil region as well as apply optimization to choose the best circle that represents the pupil area. The proposed method is applied for images from palacky university, it achieves to $100 \%$ accuracy
\end{abstract}

Copyright (C) 2018 Institute of Advanced Engineering and Science. All rights reserved.

Corresponding Author:

Ahmed Rafid Hashim,

Department of Computer Science,

College of Education for Pure Sciences-Ibn Al- Haitham, University of Baghdad, Iraq.

Email: ahmed.r.h@ihcoedu.uobaghdad.edu.iq

\section{INTRODUCTION}

Iris' eye is one of a most important human biometric that is used for persons identification and authentication. Iris segmentation is an important step in iris recognition, whereas the pupil detection is the key factor of this step. There are many studies interest in this field will be discussed such as: Daugman [1],[2],[3],[4],[5] presented method used an integrodifferential operator to localize both the inner boundary for iris (pupil detection) and outer boundary for iris. They used two arcs to detect the upper and lower eyelids. This method is sensitive to noise and does not eliminate the noise of pupil.

Wilds et al [6],[7],[8] presented a method used edge detection and hough transform to segment the eye image, this method cannot eliminate the noise of pupil and eyelashes and required a high computational cost. Tisse [9] enhanced Daugman's used a method based on reducing the computation time and solving the problem of locating the pupil center outside. With this method, one can use it without eliminating the noise produced due to eyelashes and pupil. Ma et al [10] presented a method by using hough transform after filtering the image and finding the edge points. With the use of this method, the noise of eyelashes is canceled but the pupil noise exists in the segmented area. Zhoghua Lin and Handfei Yu [11] presented a method for detecting pupil, their method consists of three stages depending on OTSU method and Hough transform. The method achieves $100 \%$ accuracy.

Imen Khanfir et al [12] presented a method based on histogram thresholding technique in order to extract pupil eye that is followed by a morphological cleaning technique to clear out the pupil binary image 
by eliminating lashes and reflection points. This method made iris localization faster by a factor of 18 . Gomai et al [13] presented a method to detect the boundary of pupil's eye based on the minimum and mean intensity of pupil, this method is able to detect and isolate pupil with high accuracy result and reduce time-consuming. Iman A. Saad et al [14] presented a new method for accurate pupil detection. The method was able to treat the bad acquisition conditions that related to low contrast or unregular brightness, where are caused by many causes, such as, the position of light sources, specular reflection, eyelashes, and eyelids. The accuracy of this method reaches to $100 \%$.

Rubel Biswas et al [15] presented a method for iris detection and recognition, the proposed method consisted of many steps segmentation, normalization, feature encoding, feature extraction, and classification. Hough Transform used for detecting the center of the pupil of an iris, the accuracy of this method reaches to 92\%. Vineet Kumar.et al [16] presented a method for iris localization, the proposed algorithm for iris localization divided into two phases which are pupil boundary detection and limbic boundary detection, they used edge map and circural hough transform for pupil boundary detection, the accuracy of this method reach to $99.7 \%$ for CASIA-Iris-Thousand (version 4.0 ) and $99.38 \%$ for CASIAIrisLamp (version 3.0) databases. Teddy et al [17] presented a method for secure smart phone using iris verification, they used wavelet packet and hamming distance for recognition, the accuracy of this method reach to $100 \%$ identification rate. In this paper, we present an approach for pupil detection based on many steps. The experimental part was applied on eye image database collected by Palacky University.

\section{THE PROPOSED METHOD}

In this method, we used a new idea for pupil detection with better accuracy. This idea depending on the differences between the intensity level (low or high level) and (chromatic or near to gray color) in the pupil and iris regions. Figure 1 illustrated the histograms for crop regions of the pupil and the iris. From the histogram of the pupil, one can see the most of the pupil region is gray or tend to gray color. This means the color difference between the (RGB) is small (the data images in the pupil is achromatic Often). The figure shows also the intensity for RGB component is low in the case of neglecting the luminous disc in the center, whereas in the iris region the intensity is high and the color deferent is clear (the data images in this Iris region is chromatic Often).

These color features isolate the iris area partially where the area of the pupil is not completely gray. It contains a slight color and the area may also contain small gray gaps. These difficulties can be overcome by binarization the pupil region, which can be achieved by use dilation and erosion of the pupil to a reach an approximate isolation for the boundary of iris. The pupil region has been fitted to Circular Hough Transform. A preliminary work area with radius $r_{w}$ is selected, It is a circular area with radius between $\left(r_{w m i n}, r_{w m a x}\right)$, through which determines the pupil boundaries as shown in Figure 2. The identification of this region provides a shortcut in time when conducting mathematical operations and computed for the entire image. First, the image is converted to a binary image $\mathrm{I}_{\mathrm{b}}$ taking into account the difference in color and intensity between the pupil and iris region.

$$
\begin{aligned}
& \text { If } c\left(i, j, k, r_{w}\right)<t_{1} \\
& c\left(i, j, k, r_{w}\right)=0
\end{aligned}
$$

Here $i, j$ being the position Coordinates, $k=1,2,3$ are the RGB component and $t_{1}$ is the greatest approximate value of intensity in a pupil area. Total differences of RGB component is:

$$
I_{t d}=d_{r g}+d_{r b}+d_{b g}
$$

Where:

$$
\begin{aligned}
& d_{r g}=a b s(r-g) \\
& d_{r b}=a b s(r-b) \\
& d_{b g}=a b s(b-g)
\end{aligned}
$$




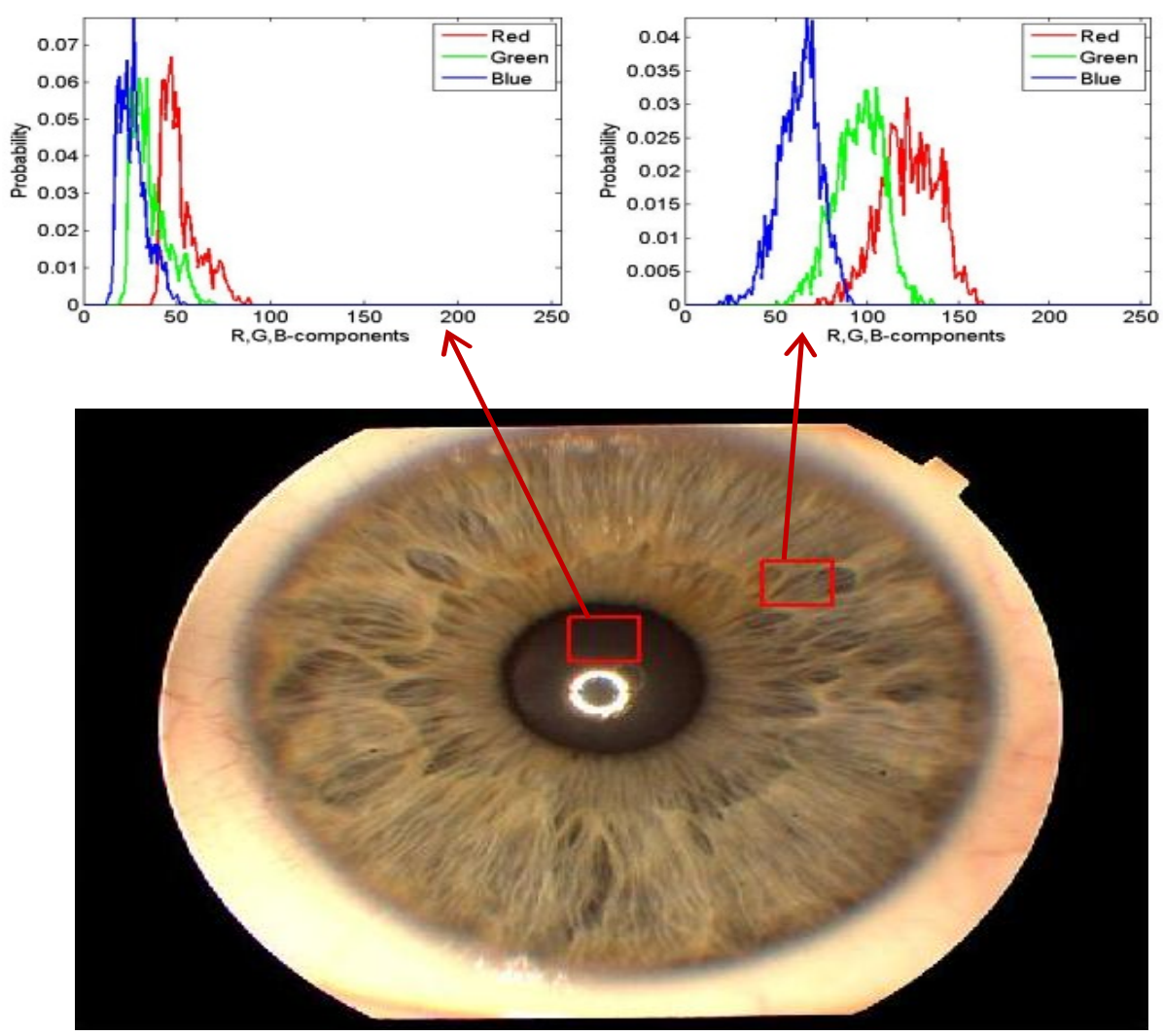

Figure 1. comparing between the histograms for pupil and iris region within certain areas.

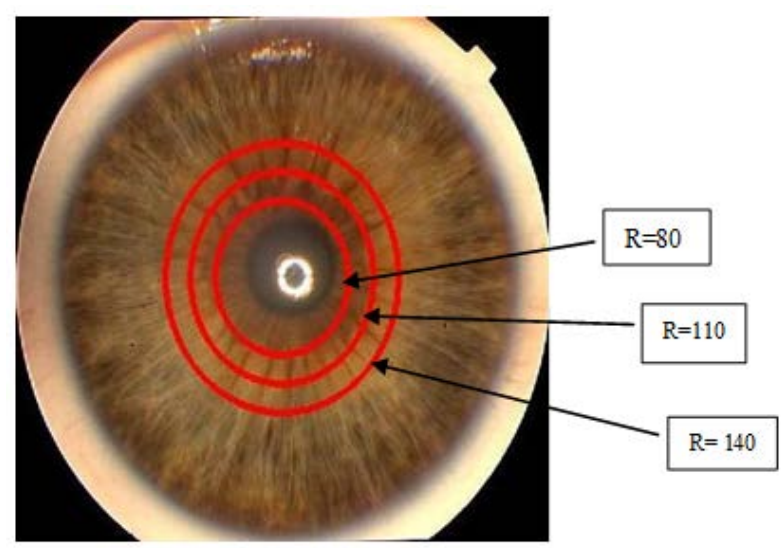

Figure 2. circle work area with different radius.

The result of this step is the region of pupil become white area as shown in Figure 3. Converting the image into a binary image depends on the threshold value $t_{2}$ where:

$$
\begin{aligned}
& t_{2 \min } \leq t_{2} \leq t_{2 \max } \\
& \text { If } I_{t d}<t_{2} \text { then } I_{b}\left(r_{w}, t_{2}\right)=0 \\
& \text { else } I_{b}\left(r_{w}, t_{2}\right)=1
\end{aligned}
$$

For the total RGB component is: 


$$
\begin{aligned}
& \mathrm{T}=\mathrm{r}+\mathrm{g}+\mathrm{b} \\
& \text { If } \mathrm{T}<\mathrm{t}_{3} \text { then } \mathrm{I}_{\mathrm{b}}\left(\mathrm{r}_{\mathrm{w}}, \mathrm{t}_{2}\right)=0
\end{aligned}
$$

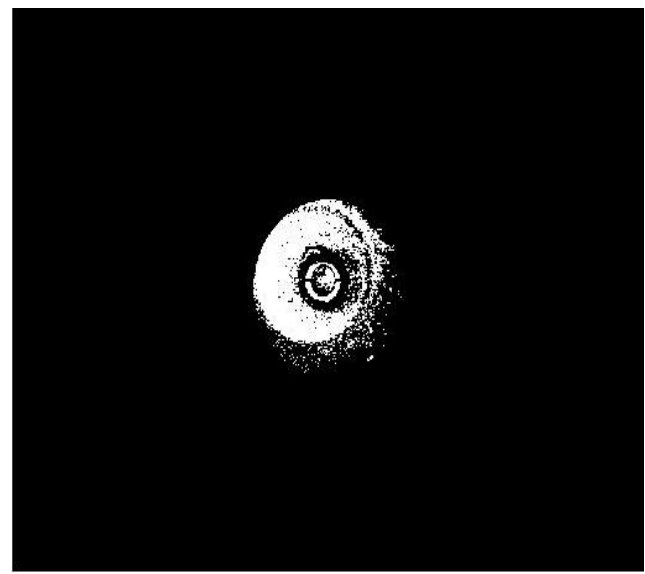

Figure 3. binary result according to color difference.

The second step is to isolate the pupil by dilating of excess areas, filling the gaps and using edge detection techniques. This is done through the following steps:

$$
I_{b d}\left(r_{w}, t_{2}\right)=I_{b}\left(r_{w}, t_{2}\right) \oplus s e
$$

Where

$$
\mathrm{se}=\left|\begin{array}{lll}
0 & 1 & 0 \\
1 & 1 & 1 \\
0 & 1 & 0
\end{array}\right|
$$

The fact that the iris region is not colored has gray-generated objects, to remove all connected components objects that have fewer area sm from the binary image, for all block region $\mathrm{cI}_{\mathrm{bd}}$ in the binary image $\mathrm{I}_{\mathrm{bd}}$ to get I bdr where:

$$
\text { IfcI }_{\text {bd }}<\text { sm then } \mathrm{cI}_{\mathrm{bd}}=0
$$

The area of the pupil is not connected because it is not absolutely gray. This resulting in a gap when converted to a gray image to connecting this area used canny edge detection and seed filling techniques to resulting.

$$
I_{b d r c f}\left(r_{w}, t_{2}\right)
$$

The result of this step shown in Figure 4. Figure 4 shows the binary image is not completely circular, thus it was used CHT that is working to complete the circular shape by input approximate radius rc as shown in Figure 5. In this study, several radii were used to get $I_{c h}\left(r_{w}, t_{2}, r_{c}\right)$, and then filling the circular region being $I_{c h f}\left(r_{w}, t_{2}, r_{c}\right)$. We will produce several circles filled with different radii , $r_{c}$.

$$
\mathrm{r}_{\mathrm{c}}=\left[\min _{\mathrm{r}_{\mathrm{c}}}, \max _{\mathrm{r}_{\mathrm{c}}}\right]
$$

To find the absolute error between $I_{b d r c f}$ and $I_{c h}$

$$
\operatorname{Er}=\left|I_{c h}\left(r_{w}, t_{2}, r_{c}\right)-I_{b d r c f}\left(r_{w}, t_{2}, r_{c}\right)\right|
$$

The best value of the parameters $r_{w}, t_{2}, r_{c}$ as shown in Figure 6 getting at: 


$$
\min _{\mathrm{w}_{\mathrm{w}}, \mathrm{t}_{2}, \mathrm{r}_{\mathrm{c}}}=0
$$

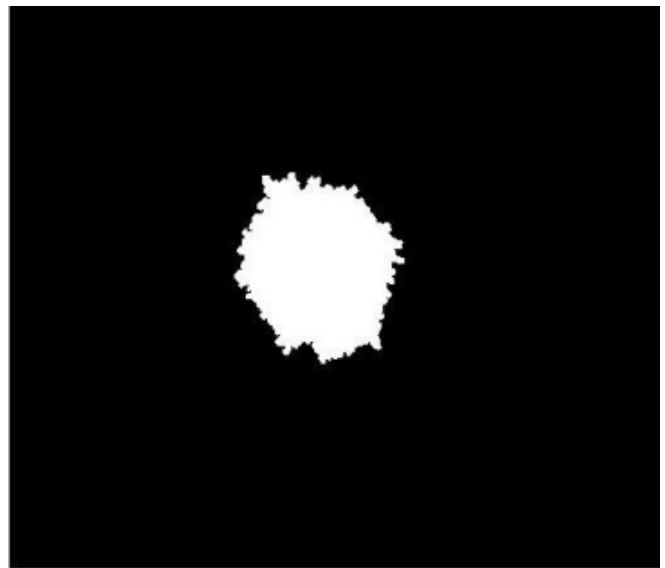

Figure 4. Binary result from removing all connected objects and seed filling the hole region.

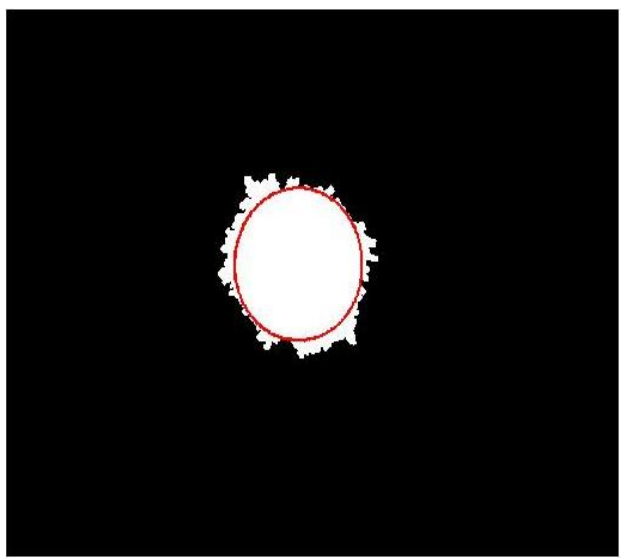

Figure 5. Circle hough transform for fitting circle.

\section{EXPERIMENTAL RESULTS}

Our method performed by using Matlab (version R2013a) on computer platform has $2.4 \mathrm{GHZ}$ core i5 processor and 4G ram. In this work we used $r_{w}=[90140]$, $t_{2}=\left[\begin{array}{lll}50 & 70\end{array}\right], t 1=50$ and $r_{c}=$ [30 to 140], The proposed method was applied to images collected by the Palacky University. The database [18] has 6* 64 iris images (i.e. $3 * 64$ left and $3 * 64$ right), the images properties are $768 * 576$ pixels, and file format is PNG. The table (1) shows the accuracy values of the pupil detection by using algorithms in [19] and [20], first value is $99.86 \%$, second $98.85 \%$ and the proposed method reach to $100 \%$ accuracy.

\section{CONCLUSION}

Pupil detection is the most important step in iris detection. In this work, we present a new method for pupil detection. The method consists of many steps beginning from reading eye image until reach to pupil detection step. The recent is the most important step where it depends on the difference in color and intensity between pupil and surrounding area. This characteristic is very useful to determine the pupil region and extract it, thus the pupil becomes has a little color and intensity than surrounding region. The new method is very successful in pupil detection, the accuracy of the method reach to $100 \%$ compare with other methods. 


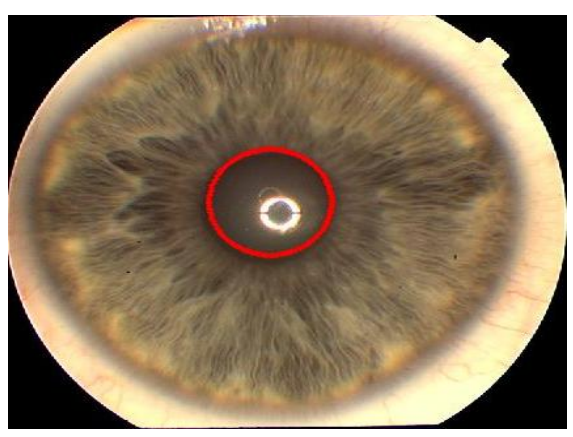

Figure 6. Final pupil detection step.

Table1. Comparsion between our Proposed Method Result and others Method

\begin{tabular}{cccc}
\hline method & A. Basit et al [19] & Ann A. Jarjes [20] & Our method \\
\hline Accuracy rate & $99.86 \%$ & $99.85 \%$ & $100 \%$ \\
\hline
\end{tabular}

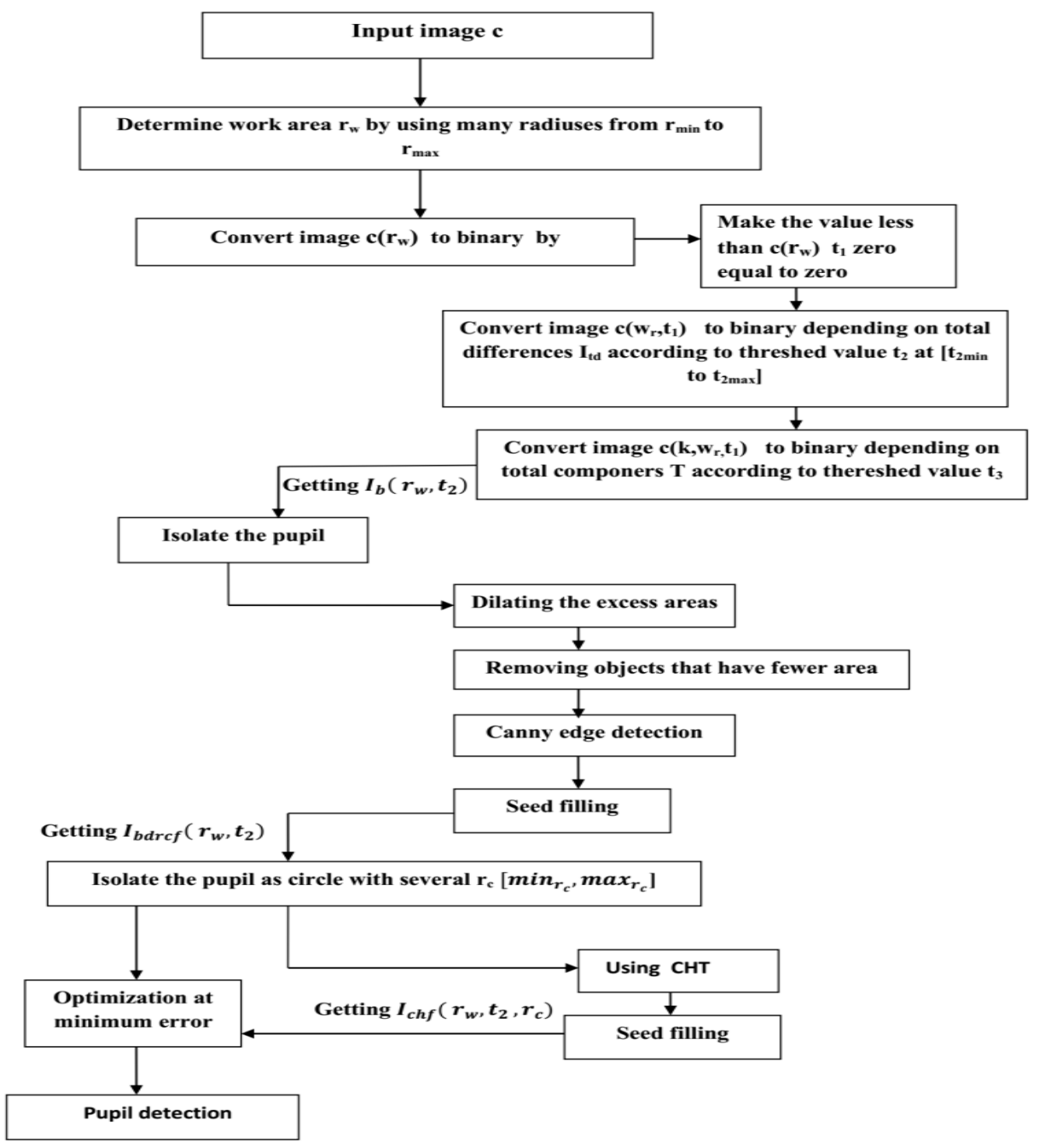

Figure 7. Flow chart of proposed method 


\section{REFERENCES}

[1] J.G. Daugman, "High confidential visual recognition by test of statistical independence”, IEEE Trans. Pattern Anal. Mach. Intell. 15 (11), pp. 1148-1161, 1993.

[2] J.G. Daugman, “The importance of being random: statistical principles of iris recognition”, Pattern Recognition 36 pp. 279-291, 2003.

[3] J. Daugman, "How iris recognition works", IEEE Transactions on Circuits and Systems for Video Technology, vol. 14, n.1, January 2004, pp. 21-30.

[4] J. Daugman, “Demodulation by complex-valued wavelets for stochastic pattern recognition”, International Journal of Wavelets, Multiresolution and Information Processing Vol. 1, n.1, pp. 1-17, 2003.

[5] J. Daugman, "Statistical richness of visual phase information: update on recognizing persons by iris patterns". International Journal of Computer Vision, 2001, 45(1), pp. 25-38.

[6] R. Wildes, “Iris recognition: an emerging biometric technology,” Proc. IEEE, vol. 85, pp. 1348-1363, Sept. 1997.

[7] R.Wildes, J.Asmuth, et al., “A Machine-vision System for Iris Recognition”, Machine Vision and Applications, Vol.9, pp.1-8, 1996.

[8] R.Wildes, J. Asmuth, et al., “Automated, non invasive iris recognition system and method,” U.S. Patent 5, pp. 572596, 1996.

[9] C. Tisse, L.Martin, L. Torres, and M. Robert. "Person identification technique using human iris recognition” In Proceedings of ICVI'02, pages 294-299, 2002.

[10] L. Ma, T. Tan, Y. Wang and D. Zhang, "Efficient iris recognition by characterizing key local variations," IEEE Trans. Image Processing, vol. 13, no. 6, June 2004.

[11] Zhonghua Lin, Hongfei Yu, "The Pupil Location Based on the OTSU Method and Hough Transform", Procedia Environmental Sciences 8 (2011) 352 - 356.

[12] Imene Khanfir Kallel, Dorra Sellami Masmoudi, Nabil Derbel, " FAST PUPIL LOCATION FOR BETTER IRIS DETECTION", 2009 6th International Multi-Conference on Systems, Signals and Devices.

[13] Gomai, A. El-Zaart, and H. Mathkour, "A New Approach for Pupil Detection in Iris Recognition System", 2010 2nd International Conference on Computer Engineering and Technology.

[14] Iman A.Saad, Loay E.George and Ahmed A.Tayyar," ACCURATE AND FAST PUPIL LOCALIZATION USING CONTRAST STRETCHING, SEED FILLING AND CIRCULAR GEOMETRICAL CONSTRAINTS", Journal of Computer Science 10 (2): 305-315, 2014.

[15] Rubel Biswas, Jia Uddin, Md. Junayed Hasan," A New Approach of Iris Detection and Recognition", International Journal of Electrical and Computer Engineering (IJECE),Vol. 7, No. 5, October 2017, pp. 2530 2536.

[16] Vineet Kumar, Abhijit Asati, Anu Gupta, " Accurate Iris Localization Using Edge Map Generation andAdaptive Circular Hough Transform for Less Constrained Iris Images ", International Journal of Electrical and Computer Engineering (IJECE), Vol. 6, No. 4, August 2016, pp. 1637 1646.

[17] Teddy Surya Gunawan, Nurul Shaieda Solihin, Malik Arman Morshidi, Mira Kartiwi, " Development of Efficient Iris Identification Algorithm using Wavelet Packets for Smartphone Application", Indonesian Journal of Electrical Engineering and Computer Science,Vol. 8, No. 2, November 2017, pp. $450 \sim 456$.

[18] https://drive.google.com/drive/folders/0B_xRqfOMgQDGbENfZFNkUTExZzA

[19] A. Basit, M. Y. Javed and S. Masood, "Non-circular Pupil Localization in Iris Images", 2008 International Conference on Emerging Technologies IEEE-ICET 2008.

[20] Ann A. Jarjes, Kuanquan Wang, Ghassan J. Mohammed, " Iris Localization: Detecting Accurate Pupil Contour and Localizing Limbus Boundary", 2010 2nd International Asia Conference on Informatics in Control, Automation and Robotics. 